\title{
Idebenone Has Distinct Effects on Mitochondrial Respiration in Cortical Astrocytes Compared to Cortical Neurons Due to Differential NQO1 Activity
}

\author{
Sausan M. Jaber, ${ }^{1,2}$ Shealinna X. Ge, ${ }^{1}{ }^{\circledR}$ Joshua L. Milstein, ${ }^{1}{ }^{\oplus}$ Jonathan W. VanRyzin, ${ }^{3,4}{ }^{\oplus}$ Jaylyn Waddell, ${ }^{5}$ and \\ ${ }^{-}$Brian M. Polster ${ }^{1,2}$ \\ ${ }^{1}$ Department of Anesthesiology and Center for Shock, Trauma and Anesthesiology Research, University of Maryland School of Medicine, Baltimore, \\ Maryland 21201, ${ }^{2}$ Department of Biochemistry and Molecular Biology, University of Maryland School of Medicine, Baltimore, Maryland 21201, \\ ${ }^{3}$ Program in Neuroscience, University of Maryland School of Medicine, Baltimore, Maryland 21201, ${ }^{4}$ Department of Pharmacology, University of \\ Maryland School of Medicine, Baltimore, Maryland 21201, and ${ }^{5}$ Department of Pediatrics, University of Maryland School of Medicine, Baltimore, \\ Maryland 21201
}

Idebenone is a synthetic quinone that on reduction in cells can bypass mitochondrial Complex I defects by donating electrons to Complex III. The drug is used clinically to treat the Complex I disease Leber's hereditary optic neuropathy (LHON), but has been less successful in clinical trials for other neurodegenerative diseases. NAD(P)H:quinone oxidoreductase 1 (NQO1) appears to be the main intracellular enzyme catalyzing idebenone reduction. However, NQO1 is not universally expressed by cells of the brain. Using primary rat cortical cells pooled from both sexes, we tested the hypotheses that the level of endogenous NQO1 activity limits the ability of neurons, but not astrocytes, to use idebenone as an electron donor to support mitochondrial respiration. We then tested the prediction that NQO1 induction by pharmacological activation of the transcription factor nuclear erythroid 2-related factor 2 (Nrf2) enables idebenone to bypass Complex I in cells with poor NQO1 expression. We found that idebenone stimulated respiration by astrocytes but reduced the respiratory capacity of neurons. Importantly, idebenone supported mitochondrial oxygen consumption in the presence of a Complex I inhibitor in astrocytes but not neurons, and this ability was reversed by inhibiting NQO1. Conversely, recombinant NQO1 delivery to neurons prevented respiratory impairment and conferred Complex I bypass activity. Nrf2 activators failed to increase NQO1 in neurons, but carnosic acid induced NQO1 in COS-7 cells that expressed little endogenous enzyme. Carnosic acid-idebenone combination treatment promoted NQO1-dependent Complex I bypass activity in these cells. Thus, combination drug strategies targeting NQO1 may promote the repurposing of idebenone for additional disorders.

Key words: carnosic acid; Catena; Complex I; LHON; Nrf2; Raxone

Significance Statement

Idebenone is used clinically to treat loss of visual acuity in Leber's hereditary optic neuropathy. Clinical trials for several additional diseases have failed. This study demonstrates a fundamental difference in the way idebenone affects mitochondrial respiration in cortical neurons compared with cortical astrocytes. Cortical neurons are unable to use idebenone as a direct mitochondrial electron donor due to NQO1 deficiency. Our results suggest that idebenone behaves as an NQO1-dependent prodrug, raising the possibility that lack of neuronal NQO1 activity has contributed to the limited efficacy of idebenone in neurodegenerative disease treatment. Combination therapy with drugs able to safely induce NQO1 in neurons, as well as other brain cell types, may be able to unlock the neuroprotective therapeutic potential of idebenone or related quinones.

Received Aug. 1, 2017; revised Apr. 18, 2020; accepted Apr. 26, 2020.

Author contributions: S.M.J. and B.M.P. designed research; S.M.J., S.X.G., and J.L.M. performed research;

S.M.J., S.X.G., J.L.M., J.W.V., J.W., and B.M.P. analyzed data; S.M.J. and B.M.P. wrote the paper.

This research was supported by National Institutes of Health Grants R01-NS-085165 and R21-NS-096538 to

B.M.P. and from the M. Jane Matjasko Endowment.

The authors declare no competing financial interests.

Correspondence should be addressed to Brian M. Polster at bpolster@som.umaryland.edu.

https://doi.org/10.1523/JNEUROSCI.1632-17.2020

Copyright $\odot 2020$ the authors

\section{Introduction}

Oxidative stress and mitochondrial bioenergetic dysfunction contribute to pathology in many disorders affecting the nervous system (Lin and Beal, 2006). Complex I of the mitochondrial electron transport chain (ETC) is particularly susceptible to impairment by oxidative stress (Danielson et al., 2011; Srinivas Bharath, 2017) and is dysfunctional in many diseases, including Leber's hereditary optic neuropathy (LHON; Chinnery et al., 
2001), Parkinson's disease (Schapira et al., 1990; Keeney et al., 2006), and Friedreich's ataxia (Heidari et al., 2009; Salehi et al., 2014). Complex I shuttles electrons to ubiquinone, also known as Coenzyme $\mathrm{Q}_{10}(\mathrm{CoQ})$, which subsequently carries electrons downstream to Complex III (Nicholls and Ferguson, 2013). Complex I also pumps protons out of the matrix to help generate the proton motive force required for ATP production.

$\mathrm{CoQ}$, in addition to its important role as an electron carrier, is an antioxidant abundantly found in cellular membranes (James et al., 2004). However, CoQ displays poor bioavailability (Tomono et al., 1986), hindering its therapeutic use. Idebenone contains the same quinone moiety as CoQ but has a shorter, less hydrophobic tail that increases solubility and improves bioavailability (Gueven et al., 2015). Idebenone-mediated neuroprotection was observed in various in vitro and in vivo models of neuronal injury, including oxidative stress (Ratan et al., 1994), stroke (Nagaoka et al., 1989), Alzheimer's disease (Yamada et al., 1999), and LHON (Heitz et al., 2012).

Idebenone was recently approved in Europe for the treatment of LHON and mitigates loss of visual acuity (Klopstock et al., 2011; Lyseng-Williamson, 2016). However, despite initially positive reports (Gutzmann and Hadler, 1998; Gutzmann et al., 2002), idebenone ultimately failed in clinical trials for Alzheimer's disease (Thal et al., 2003) and had mixed results with regard to neurologic symptoms in patients with Friedreich's ataxia (Di Prospero et al., 2007a; Lynch et al., 2010; Meier et al., 2012). Idebenone remains in several active clinical trials, including for multiple sclerosis and Duchenne muscular dystrophy. A better understanding of how idebenone affects the bioenergetics of different types of brain cells may help improve on its clinical efficacy for LHON treatment and promote success in ongoing disease trials. Therefore, our objective was to elucidate the mitochondrial bioenergetic effects of idebenone on neurons and astrocytes.

$\mathrm{NAD}(\mathrm{P}) \mathrm{H}$ :quinone oxidoreductase 1 (NQO1) is an enzyme regulated by the nuclear erythroid 2-related factor 2 (Nrf2)-antioxidant response element (ARE) transcriptional antioxidant response pathway (Li and Kong, 2009; Dinkova-Kostova and Talalay, 2010) that is able to reduce idebenone to idebenol via cytoplasmic NAD(P)H oxidation (Haefeli et al., 2011). Idebenol can then transfer electrons to Complex III like CoQ, bypassing a need for upstream electron transport chain components, including Complex I. The relative ability of idebenone to rescue ATP levels in the presence of a Complex I inhibitor across several cell types correlated well with the level of NQO1 mRNA expression (Haefeli et al., 2011). NQO1 immunoreactivity was detected in mouse retinal ganglion cells, the main degenerating cell type in LHON (Heitz et al., 2012), coinciding with the clinical efficacy of idebenone in that disease. However, in the brain, NQO1 is predominately expressed by astrocytes, with little to no expression in cortical neurons (Schultzberg et al., 1988; Stringer et al., 2004; Bell et al., 2015). We therefore tested the hypothesis that deficient NQO1 expression in cortical neurons relative to astrocytes is limiting for the ability of idebenone to stimulate Complex I-independent respiration.

Our results support the hypothesis that idebenone and related quinones behave as prodrugs requiring NQO1 activity and additionally show that idebenone impairs neuronal mitochondrial respiratory capacity by inhibiting Complex I-dependent respiration. We predicted that pharmacological stimulation of NQO1 expression by activators of the Nrf2 pathway would overcome this idebenone-mediated respiratory inhibition. We provide proof-of-principle that the induction of NQO1 in cells with poor
NQO1 expression promotes the ability of idebenone to act as a direct respiratory chain electron donor.

\section{Materials and Methods}

Astrocyte culture. All animal procedures were approved by the University of Maryland Institutional Animal Care and Use Committee and were consistent with the National Institutes of Health Guide for the Care and Use of Laboratory Animals. Cortical astrocytes were isolated from postnatal day 1 Sprague Dawley rat pups as described previously (Zielke et al., 1990; Danilov and Fiskum, 2008). Following killing of the pups, cortices were separated from midbrain and meninges, and mechanically homogenized in Eagle's minimal essential medium (EMEM; Quality Biological) containing 10\% fetal bovine serum (FBS; Sigma-Aldrich), $1 \%$ nonessential amino acids (NEAAs; Lonza), and $100 \mu \mathrm{g} / \mathrm{ml}$ gentamicin (Quality Biological). The homogenate was vortexed for $1 \mathrm{~min}$ and then filtered through a $70 \mu \mathrm{m}$ mesh filter. Cells were plated in EMEM containing 10\% FBS and 1\% NEAAs into 150 $\mathrm{cm}^{2}$ vented flasks (Sigma-Aldrich) and maintained in a humidified incubator in $95 \%$ air $/ 5 \% \mathrm{CO}_{2}$ at $37^{\circ} \mathrm{C}$. Cultures were passaged after $18 \mathrm{~d}$ in vitro (DIV) using TrypLE Express (Thermo Fisher Scientific) and used for experiments between DIV 20 and DIV 27. This method yields a $>93 \%$ astrocyte-enriched culture, with $<5 \%$ microglia and $<2 \%$ oligodendrocytes or neurons (Danilov and Fiskum, 2008). Astrocytes were seeded at least $2 \mathrm{~d}$ before measurement on Seahorse XF24 V7-PS Cell Culture Microplates (Agilent Technologies) at a density of 60,000 cells/well. Cells derived from both males and females were used for experiments.

Neuron culture. Primary cortical neurons were prepared from embryonic day 18 Sprague Dawley male and female pooled rat embryos (one litter per preparation, $\sim 12$ embryos) as described previously (Yakovlev et al., 2001; Stoica et al., 2005; Laird et al., 2013). Briefly, XF24 V7-PS cell plates were coated using poly-D-lysine hydrobromide $\left(0.1 \mathrm{mg} / \mathrm{ml}\right.$; Sigma-Aldrich), incubated at $37^{3} \mathrm{C}$ for at least $4 \mathrm{~h}$, and washed once with sterile water. Isolated cortices were digested in trypsin and treated with DNase I (Sigma-Aldrich). Cell suspension was filtered through a $70 \mu \mathrm{m}$ mesh filter and plated in Neurobasal media containing either B27 (Thermo Fisher Scientific) or Gem21 supplement (Gemini Bio-Products), $10 \%$ FBS, and $100 \mathrm{IU} / \mathrm{ml}$ penicillin with $100 \mathrm{mg} / \mathrm{ml}$ streptomycin (Gemini Bio-Products). Neurons were plated at 60,000-80,000 cells/well for respiration measurements. After $2 \mathrm{~h}$, medium was replaced with Neurobasal media containing B27 or Gem21 without FBS. Cytosine arabinofuranoside ( $5 \mu \mathrm{M}$; Sigma-Aldrich) was added on DIV 4 to inhibit glial proliferation. Additional FBS-free Neurobasal media equal to $50 \%$ of the existing total media volume was added on DIV 6. This method yields a $>95 \%$ neuron-enriched culture (Laird et al., 2013). Neurons were used for experiments between DIV 10 and DIV 14.

COS-7 cell culture. Subjects included the COS-7 cell line (catalog \#605470/p532_COS-7, CLS; RRID:CVCL_0224. COS-7 cells were maintained in DMEM (Corning) containing $10 \%$ FBS and $100 \mathrm{IU} / \mathrm{ml}$ penicillin with $100 \mathrm{mg} / \mathrm{ml}$ streptomycin. COS-7 cells were passaged using TrypLE Express and were plated at 20,000 cells/well for respiration studies. Cells were used after $72 \mathrm{~h}$ of carnosic acid $(3 \mu \mathrm{M})$ or vehicle treatment.

Cellular respirometry. Cellular respiration was measured using a Seahorse XF24 Extracellular Flux Analyzer (Agilent Technologies) as described (Wu et al., 2007; Clerc and Polster, 2012). Assay medium was artificial CSF (aCSF) consisting of $120 \mathrm{~mm} \mathrm{NaCl}, 3.5 \mathrm{~mm} \mathrm{KCl}, 1.3 \mathrm{~mm}$ $\mathrm{CaCl}_{2}, 0.4 \mathrm{~mm} \mathrm{KH} \mathrm{PO}_{4}, 1 \mathrm{~mm} \mathrm{MgCl}, 5 \mathrm{~mm} \mathrm{HEPES}, 4 \mathrm{mg} / \mathrm{ml}$ fatty-acid

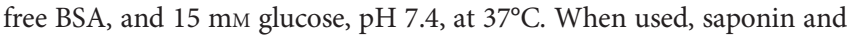
sodium pyruvate were always prepared fresh from powder. Before assays, cell culture medium was removed, cells were washed once in $1 \mathrm{ml}$ $\mathrm{aCSF}$, and then cells were incubated in $675 \mu \mathrm{l}$ of aCSF in a $\mathrm{CO}_{2}$-free incubator $\left(37^{\circ} \mathrm{C}\right)$ for $45 \mathrm{~min}$. Drug treatments were diluted in $75 \mu \mathrm{l}$ of aCSF and added to the XF24 Seahorse assay cartridge (Agilent Technologies) at $10 \times, 11 \times, 12 \times$, or $13 \times$ the final working concentration for ports A-D, respectively. Three measurements of oxygen consumption rate (OCR) were made before any drug additions to establish a baseline. Because there are several factors that contribute to OCR 
variation of primary cell cultures, including variability in cell counting, cell distribution in the wells relative to where the $\mathrm{O}_{2}$ sensor measures, and cell survivability (Gerencser et al., 2009; Clerc et al., 2013), OCRs are presented throughout as a percentage of basal or maximal respiration. We showed previously by power analysis that elimination of technical variability by normalization reduces the number of replicates, and hence, the number of animals, needed to observe a $20 \%$ difference in respiration in response to acute drug treatment (Schuh et al., 2011).

Enzymatically active recombinant human NQO1 (catalog \#D1315, Sigma-Aldrich) and NADPH substrate (Sigma-Aldrich) were delivered to neurons via saponin permeabilization. In permeabilized cell experiments, saponin was used at low enough concentrations to permeabilize cholesterol-containing membranes such as the plasma membrane while leaving the cholesterol-poor mitochondrial inner and outer membranes intact (Clerc and Polster, 2012). Saponin $(25 \mu \mathrm{g} / \mathrm{ml})$ was added with the Complex I-linked substrates pyruvate and malate ( $5 \mathrm{~mm}$ each), the ATP synthase substrates ADP $(1 \mathrm{~mm})$ and phosphate $\left(3.6 \mathrm{~mm} \mathrm{KH}_{2} \mathrm{PO}_{4}\right)$ to stimulate respiration, and the calcium chelator EGTA $(5 \mathrm{~mm})$ to reduce calcium to a normal cytoplasmic level $(\sim 100 \mathrm{nM})$. Subsequent injection of succinate (5 mM) was used to support Complex II-dependent respiration in permeabilized cells.

Antibodies. The following antibodies were used: primary rabbit polyclonal anti-NQO1 (catalog \#N5288, Sigma-Aldrich; RRID:AB_1841045); primary rabbit monoclonal anti- $\beta$-glyceraldehyde-3-phosphate dehydrogenase (GAPDH; catalog \# 2118, Cell Signaling Technology; RRID: AB_561053); and secondary goat anti-rabbit IgG (H + L) HRP antibody (catalog \#65-6120, Thermo Fisher Scientific; RRID:AB_2533967).

Western blot. Cells were hypotonically lysed in $25 \mathrm{~mm}$ Tris- $\mathrm{HCl}$ and 1:200 protease inhibitor cocktail (EMD Millipore). Novex Tris-Glycine SDS Sample Buffer and Novex NuPAGE Sample Reducing Agent (Thermo Fisher Scientific) were added, and the samples were heated for $10 \mathrm{~min}$ at $95^{\circ} \mathrm{C}$. Samples were then centrifuged for $10 \mathrm{~min}$ at $14,000 \mathrm{~g}$, and protein concentrations were measured using a NanoDrop ND-1000 (Thermo Fisher Scientific). Novex WedgeWell Tris-Glycine gels (Thermo Fisher Scientific) were loaded with $10 \mu \mathrm{g}$ of protein per well. Transfer to a polyvinylidene difluoride membrane was performed using the Trans-Blot Transfer System (Bio-Rad). Membranes were probed overnight at $4^{\circ} \mathrm{C}$ using the NQO1 and GAPDH antibodies described above at 1:10,000 dilution. Blots were then washed and treated with horseradish peroxidase-conjugated secondary anti-rabbit antibody (Thermo Fisher Scientific). Bands were visualized using SuperSignal West Pico ECL (Thermo Fisher Scientific) and imaged using the BioRad ChemiDoc XRS. Densitometric quantification of band intensities was performed using ImageJ software (National Institutes of Health).

Experimental design and statistical analysis. NQO1 protein levels were analyzed by Student's $t$ test. OCR data from the experiment evaluating the effects of idebenone on neurons compared with astrocytes were analyzed using a two-way ANOVA, with cell type and idebenone treatment as factors. OCR data from the experiment evaluating the effect of idebenone treatment on astrocytes plus and minus concomitant dicoumarol treatment were analyzed using two-way ANOVA, with idebenone and dicoumarol treatment as factors. OCR data from the experiment evaluating the effect of recombinant NQO1 treatment on astrocytes plus and minus idebenone were analyzed using two-way ANOVA, with idebenone treatment and the enzyme cotreatment as factors. OCR data from the experiment evaluating the effect of idebenone on carnosic acidpretreated versus vehicle-pretreated cells were analyzed using a two-way ANOVA, with the pretreatment and the subsequent treatment as factors. All other OCR data were analyzed by one-way ANOVA. Statistical analyses were performed using GraphPad Prism (GraphPad Software). Significance level was set at $p<0.05$. When a significant overall difference was detected, Tukey's post hoc analysis was used to compare individual groups and $p$ values for comparisons of interest are reported. Data from two to three wells (technical replicates) per experiment were averaged to obtain one biological replicate from an independent cell culture preparation. Biological replicates are reported as the mean \pm SE in all figures.

\section{Results}

\section{Idebenone differentially affects oxygen consumption by neurons compared with astrocytes}

We previously characterized the respiratory characteristics of primary rat cortical neurons (Clerc and Polster, 2012; Clerc et al., 2013) and astrocytes (Jaber et al., 2018), including responses to oligomycin and uncoupler. Here, the effects of idebenone on cellular basal respiration and the respiratory capacity of these cells were investigated using a Seahorse XF24 Extracellular Flux analyzer. Oligomycin was not included in these experiments because it blunts the response to uncoupler, underestimating respiratory capacity (Ruas et al., 2016). We found a significant idebenone by cell type interaction in basal and maximal oxygen consumption rate, driven by the different responses of neurons and astrocytes to idebenone treatment $\left(F_{(4,37)}=11.74, p<0.001\right.$; and $F_{(4,33)}=$ 24.28, $p<0.001$, respectively; Fig. 1 , compare $A$, neurons, $B$, astrocytes). OCR by cortical neurons was not altered in response to idebenone injection (all post hoc comparisons, $p \geq 0.998$; Fig. $1 A, C)$, but maximal respiration, measured after the addition of the uncoupler 2,4-dinitrophenol (DNP) and excess mitochondrial substrate, was inhibited in a dose-dependent manner $[p=0.0359$ for $10 \mu \mathrm{M}$ idebenone compared with control $(0 \mu \mathrm{M}$ idebenone); $p<0.001$ for all other post hoc idebenone to control comparisons; Fig. 1A,D]. Idebenone can donate electrons to Complex III of the mitochondrial electron transport chain in some cell types, bypassing Complex I (Haefeli et al., 2011; Giorgio et al., 2012). There was a significant idebenone by cell type interaction on the measured OCR subsequent to addition of the Complex I inhibitor piericidin $\mathrm{A}\left(F_{(4,33)}=22.28, p<0.001\right)$, which was driven by an effect of idebenone in astrocytes (Fig. $1 B$; discussed below) that was absent in neurons. In neurons, piericidin A injection after idebenone and DNP were added impaired respiration to the same extent as in cells that received no prior idebenone treatment (all post hoc comparisons, $p>0.999$; Fig. $1 A, E)$, suggesting that idebenone does not support electron flux directly to Complex III in neurons.

In contrast to neurons, primary rat cortical astrocytes treated with idebenone demonstrated a rapid, dose-dependent stimulation of basal respiration compared with control $(p=0.288$, $0.0036,0.0015$, and $<0.001$ for $10,20,40$, and $80 \mu \mathrm{M}$ idebenone, respectively; Fig. $1 B, C$ ), and no effect on maximal $\mathrm{O}_{2}$ consumption rate was observed (all post hoc comparisons, $p>0.848$; Fig. $1 B, D)$. Additionally, a dose-dependent rescue of piericidin Aimpaired respiration was seen with increasing concentrations of idebenone $(p<0.001$ for 20,40 , or $80 \mu \mathrm{M}$ idebenone compared with control; and for $40 \mu \mathrm{M}$ compared with $80 \mu \mathrm{M}$ idebenone; Fig. $1 B, E, F)$, suggesting the bypass of inhibited Complex I. Oxygen consumption in the presence of piericidin A and idebenone was blocked by injection of the Complex III inhibitor antimycin A (Fig. $1 B, F$ ), indicating that Complex III and downstream components of the ETC are required for the idebenone rescue of respiration in astrocytes.

\section{Idebenone-stimulated respiration in astrocytes is coupled to ATP synthesis}

To test whether uncoupling contributes to idebenone-stimulated OCR in astrocytes, cells were treated with the ATP synthase inhibitor oligomycin after the addition of idebenone (Fig. 2A). Oligomycin suppresses oxygen consumption due to ATP synthesis (Brand and Nicholls, 2011). Residual OCR is primarily due to proton leak across the mitochondrial inner membrane. The oligomycin sensitivity of respiration can therefore be used to 

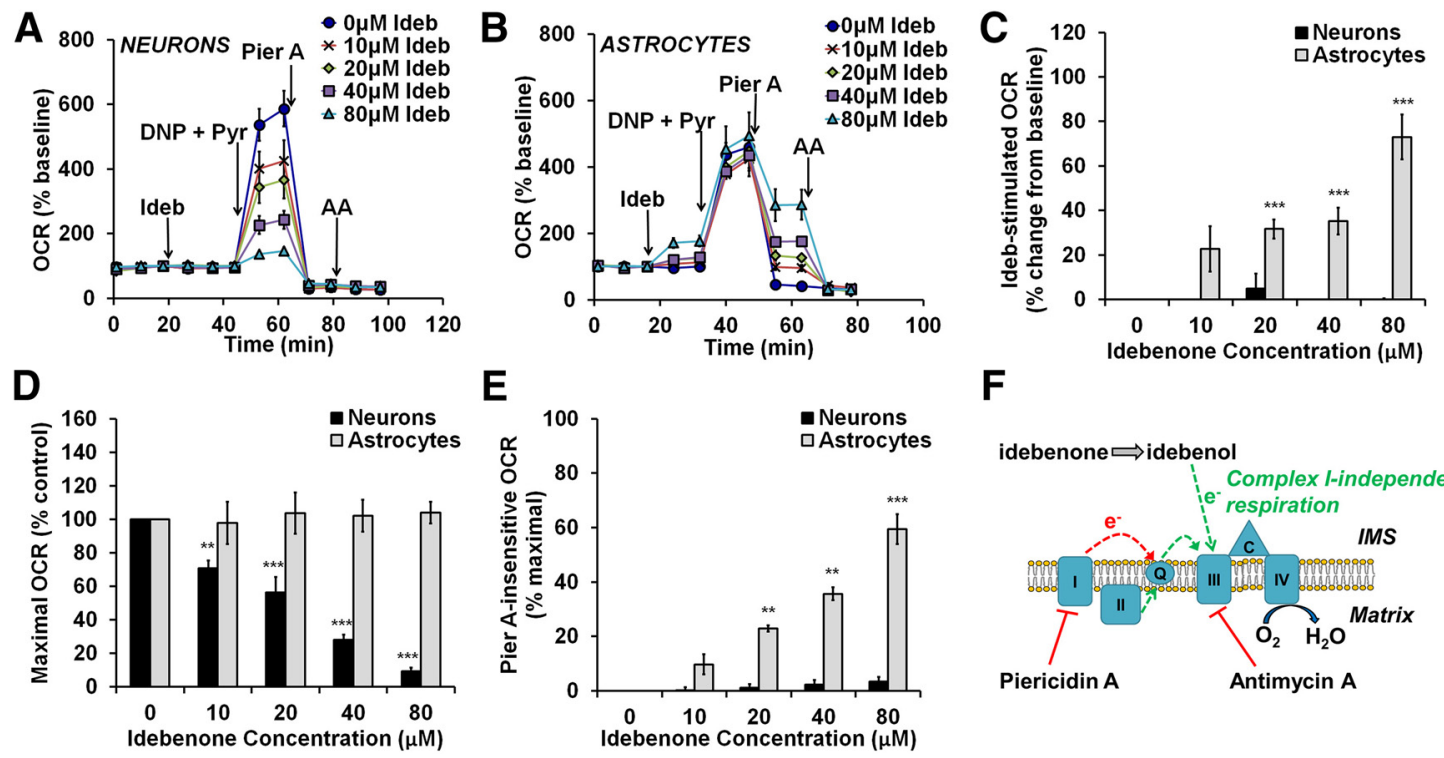

$\mathbf{F}$

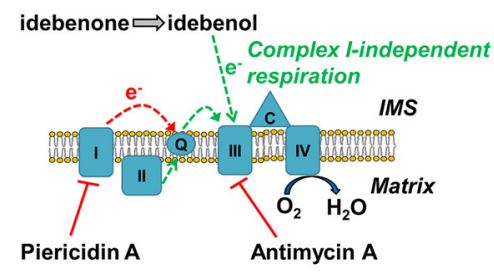

Figure 1. Idebenone stimulates Complex l-independent respiration in astrocytes but inhibits maximal respiration in neurons. $A, O C R$ measurements from primary rat cortical neurons. Idebenone (Ideb) was added at 0 (ethanol vehicle) to $80 \mu \mathrm{m}$, as indicated. The uncoupler 2,4-dinitrophenol (DNP; $200 \mu \mathrm{m}$ ) was then added in the presence of pyruvate (Pyr; $10 \mathrm{~mm}$ ) to induce maximal OCR. Piericidin A (Pier A; $100 \mathrm{~nm}$ ) and Antimycin A (AA; $1 \mu \mathrm{m}$ ) were added to inhibit Complex I and Complex III, respectively. $\boldsymbol{B}$, The same experiment described in $\boldsymbol{A}$, but using primary rat cortical astrocytes in place of neurons and $150 \mu \mathrm{m}$ DNP rather than $200 \mu \mathrm{m}$. Data in $\boldsymbol{A}$ are the mean \pm SE from three to five independent experiments, and data in $\boldsymbol{B}$ are mean \pm SE from three to seven independent experiments. $\boldsymbol{C}-\boldsymbol{E}$, Bar graph representations of data from $\boldsymbol{A}$ and $\boldsymbol{B}$. $\boldsymbol{C}, 0 C R$ after idebenone injection relative to basal $0 C$. Some values were slightly negative compared with baseline but are depicted as zero since OCR stimulation is plotted. $D, O C R$ after injection of DNP plus pyruvate ("Maximal OCR") with increasing idebenone concentration relative to vehicle (0 $\mu$ m idebenone). $\boldsymbol{E}, 0 C R$ after injection of piericidin $A$ with increasing idebenone concentration, relative to maximal $0 C R$ of control ( $0 \mu$ idebenone). $\boldsymbol{F}$, Schematic diagram illustrating the ability of idebenone to support Complex I-independent respiration. The Complex I inhibitor piericidin A suppresses the flow of electrons (e-, shown in red) from Complex I to CoQ (Q). Idebenone, when reduced to idebenol, transfers e- to Complex III, bypassing the block at Complex I. Electron entry through Complex II (green arrows) can also bypass Complex I inhibition (Fig. 4). IMS, Intermembrane space. ${ }^{* *} p<0.01$ relative to vehicle control; ${ }^{* *}<0.001$ relative to control; two-way ANOVA followed by Tukey's post hoc test.

estimate the amount by which electron transport is coupled to ATP synthesis compared with that uncoupled due to proton leak (Brand and Nicholls, 2011). Oligomycin suppressed OCR to the same level in the absence and presence of idebenone (one-way ANOVA: $F_{(4,14)}=$ $0.429, p=0.786$; Fig. $2 B$ ). Because the enhanced OCR on idebenone addition was abolished by oligomycin, idebenonestimulated OCR is therefore coupled to ATP synthesis, consistent with the ability of idebenone to rescue ATP levels when Complex I is inhibited (Haefeli et al., 2011; Giorgio et al., 2012). The stimulation of basal respiration by idebenone suggests that astrocyte respiration may be limited by mitochondrial substrate supply when glucose is the lone biofuel. Consistent with this possibility, we found that the acute addition of the cell-permeable Complex I substrate pyruvate stimulated OCR to $161 \pm 8 \%$ of the baseline rate (mean $\pm \mathrm{SD} ; n=5$ wells; data not shown).

Inhibition of NQO1 in astrocytes reverses the ability of idebenone to promote Complex I-independent respiration The enzyme NQO1 reduces idebenone to idebenol in the presence of $\mathrm{NAD}(\mathrm{P}) \mathrm{H}$, altering bioactivity (Haefeli et al., 2011). We quantified NQO1 protein expression levels in whole-cell lysates of primary rat cortical astrocytes and neurons. As predicted by the reported expression pattern of NQO1 in the brain (Schultzberg et al., 1988; Stringer et al., 2004; Bell et al., 2015), astrocytes displayed abundant expression of NQO1, while NQO1 immunoreactivity was essentially absent in neurons (Fig. $\left.3 A ; t_{(4)}=22.96, p<0.001\right)$. To test whether NQO1 activity is required for the direct electron transfer activity of idebenone in astrocytes, idebenone was added in the presence or absence of the NQO1 inhibitor dicoumarol (Fig. 3B). Dicoumarol was used at concentrations $(20-80 \mu \mathrm{M})$ that did not induce uncoupling or respiratory inhibition (Fig. 3B; data not shown). There was a significant idebenone-dicoumarol treatment interaction on maximal OCR $\left(F_{(3,27)}=4.399, p=0.0121\right)$ and on OCR in the presence of the Complex I inhibitor piericidin $\mathrm{A}\left(F_{(3,27)}=10.31\right.$, $p=0.0001$ ), driven by the abilities of dicoumarol to unmask and reverse the effects of idebenone on these parameters, respectively. In the presence of dicoumarol ( 40 or $80 \mu \mathrm{M}), 80 \mu \mathrm{m}$ idebenone 

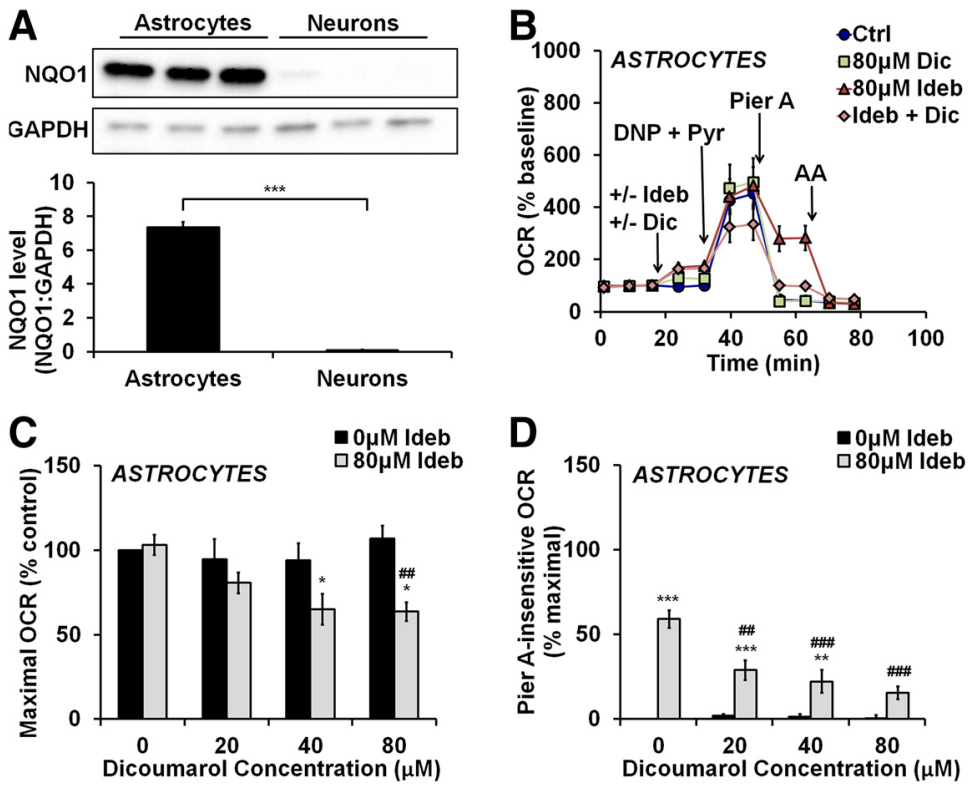

Figure 3. The ability of idebenone to support Complex I-independent respiration in astrocytes is reversed by NQ01 inhibitor. A, Immunoblot for NQ01 in primary rat cortical astrocytes and neurons, along with immunodetection of GAPDH as a loading control. NQ01 levels are expressed as a ratio to GAPDH and are the mean \pm SE from three independent experiments. ${ }^{* * *} p<0.001$, Student's $t$ test. $\boldsymbol{B}, 0$ CR measurements from primary rat cortical astrocytes. The initial injection contained vehicle, dicoumarol (Dic; $80 \mu \mathrm{m}$ ), idebenone (Ideb; $80 \mu \mathrm{M}$ ), or the two drugs combined. 2,4-dinitrophenol plus pyruvate (DNP + Pyr) was added as in Figure 1, followed by piericidin $A$ (Pier $A ; 500 \mathrm{nM})$, and then antimycin $A(A A ; 1 \mu \mathrm{M})$. Data are the mean \pm SE from three to seven independent experiments. $\boldsymbol{C}, \boldsymbol{D}$, Bar graph representations of data in $\boldsymbol{B}$ and including additional dicoumarol treatments not depicted in $\boldsymbol{B}$ for clarity. $\boldsymbol{C}$, Maximal OCR with and without idebenone and increasing concentrations of dicoumarol, expressed as a percentage of control (no dicoumarol or idebenone). $\boldsymbol{D}, \mathrm{OCR}$ after injection of piericidin $A$ with and without idebenone and increasing concentrations of dicoumarol, relative to maximal OCR of control (no dicoumarol or idebenone). ${ }^{*} p<0.05$ relative to vehicle control; ${ }^{* *} p<0.01$ relative to control; ${ }^{* * *} p<0.001$ relative to control; \#\#p $<0.01$ relative to $80 \mu \mathrm{m}$ idebenone alone; \#\# $<0.001$ relative to $80 \mu \mathrm{m}$ idebenone alone, twoway ANOVA followed by Tukey's post hoc test.

significantly impaired maximal respiration compared with control $(p=0.0144$ and $p=0.0100$ for 40 and $80 \mu \mathrm{M}$ dicoumarol, respectively; Fig. $3 C$ ), and piericidin A-insensitive respiration was reduced $(p<0.001$ for both 40 and $80 \mu \mathrm{M}$ dicoumarol added with $80 \mu \mathrm{M}$ idebenone compared with $80 \mu \mathrm{M}$ idebenone alone; Fig. 3D). Thus, the addition of NQO1 inhibitor to astrocytes recapitulates the bioenergetic effects of idebenone on neurons that lack NQO1.

\section{Idebenone inhibits Complex I-dependent neuronal respiration}

Next, we sought to determine the site of respiratory inhibition by idebenone in neurons (Fig. $1 A, D$ ), with the goal of reversing inhibition. Neurons were permeabilized by saponin to deliver substrates for specific ETC complexes. Saponin, when titrated carefully, permeabilizes the plasma membrane without affecting the integrity of mitochondrial membranes (Safiulina et al., 2004; Clerc and Polster, 2012). When idebenone was added to permeabilized neurons exposed to Complex I-linked substrates, we found that idebenone impaired ADP-stimulated oxygen consumption in a dose-dependent manner (Fig. $4 A, B$; $\left.F_{(4,10)}=11.87, p<0.001\right)$. The Complex II substrate succinate rescued the majority of the respiratory impairment by idebenone, suggesting that idebenone inhibits the electron transport chain at or upstream of Complex I (Fig. $4 A, C$ ). Following succinate treatment, we still detected a minor respiratory impairment across all concentrations of idebenone $\left(F_{(4,10)}=3.495\right.$, $p=0.0494)$; however, none of the post hoc comparisons were significant.

\section{Recombinant NQO1 and NADPH reverse respiratory inhibition by idebenone}

If the absence of NQO1 expression in neurons accounts for the distinct effects of idebenone on respiration relative to astrocytes, then supplementation of neurons with exogenous NQO1 and its substrate NADPH should prevent respiratory inhibition by idebenone and induce an astrocyte-like phenotype. Primary cortical neurons were permeabilized and provided with Complex I substrates in the presence or absence of recombinant human NQO1 and NADPH (Fig. 5A). We detected a significant idebenoneenzyme treatment interaction on both ADP-stimulated OCR $\left(F_{(3,30)}=4.213\right.$, $p=0.0134)$ and piericidin A-insensitive OCR $\left(F_{(3,22)}=3.985, p=0.0208\right)$, driven by the ability of recombinant NQO1+ $\mathrm{NADPH}$ to rescue the inhibitory effect of idebenone on respiration and allow it to increase OCR in the presence of Complex I inhibitor, respectively. As shown earlier, ADP-stimulated oxygen consumption was inhibited after the addition of idebenone alone (Fig. 5A; $p<0.001)$. Respiratory inhibition was reversed when idebenone was added in the presence of both exogenous NQO1 and its substrate, NADPH $(p=0.0014)$, but not when NQO1 alone $(p=0.975)$ or NADPH alone $(p>0.999)$ was added (Fig. $5 A, B$ ). The joint addition of idebenone, NQO1, and NADPH also induced a significant increase in piericidin A-insensitive oxygen consumption $(p<0.001$; Fig. $5 A, C$ ) that was inhibited by antimycin A (Fig. $5 A$ ). Collectively, results indicate that idebenone behaves as a prodrug that requires reduction by NQO1 to acquire Complex I bypass activity.

\section{The Complex I bypass activity of two natural naphthoquinones is also absent in neurons}

Next, we sought to determine whether other quinones with Complex I bypass potential would display bypass activity in cortical neurons lacking NQO1 expression. Like idebenone, $\alpha$-tocopherolquinone [ $\alpha$-TPQ (also called EPI-A0001)] was tested in a clinical trial for the treatment of Friedreich's ataxia (Lynch et al., 2012). However, $\alpha$-TPQ did not significantly affect the basal, maximal, or piericidin A-insensitive respiration of either neurons (one-way ANOVA: $F_{(4,10)}=0.3518, p=0.8371 ; F_{(4,10)}=$ $0.3694, p=0.8252$; and $F_{(4,10)}=1.263, p=0.347$, respectively; Fig. $6 A$ ) or astrocytes (one-way ANOVA: $F_{(4,15)}=0.1654, p=0.9527$; $F_{(4,15)}=0.6166, p=0.6574$; and $F_{(4,15)}=1.598, p=0.2263$, respectively; Fig. $6 B$ ). Thus, $\alpha$-TPQ not only has no Complex I bypass activity in neurons, but also lacks idebenone-like bypass activity in astroyctes.

Two naphthoquinones, 2-methoxy-1,4-naphthoquinone (MNQ) and 2-methoxy-3-methyl-1,4-naphthoquinone (Me-MNQ), were shown to stimulate respiration in a Complex I-deficient myoblast cell line (Vafai et al., 2016). We found that MNQ dose-dependently increased basal respiration (one-way ANOVA, $F_{(4,17)}=$ 

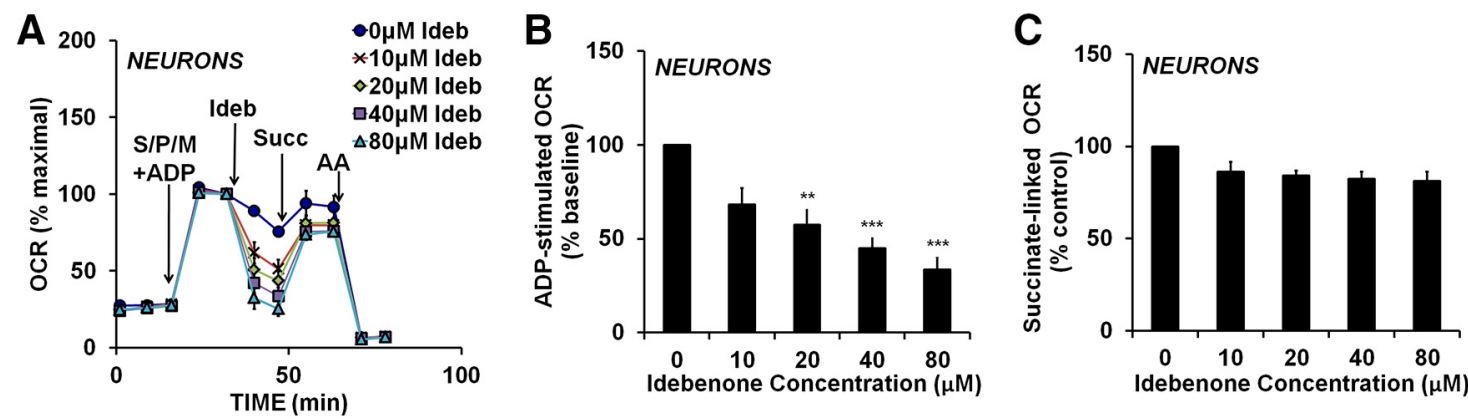

Figure 4. Idebenone inhibits Complex I-dependent respiration, but not the downstream electron transport chain. $A, O C R$ measurements from cortical neurons. Neurons were first permeabilized by the injection of saponin in the presence of the Complex I-linked substrates pyruvate and malate (collectively abbreviated as S/P/M), as described in Materials and Methods, with ADP (1 $\mathrm{mm}$ ) added to stimulate respiration. Idebenone (Ideb) was then added at a concentration of 0 (vehicle) to $80 \mu \mathrm{m}$, as indicated. Subsequent additions were succinate (Succ; $5 \mathrm{~mm}$ ) followed by antimycin $\mathrm{A}(\mathrm{AA} ; 1 \mu \mathrm{M})$. OCR measurements are normalized to the point just before idebenone/vehicle addition (ADP-stimulated baseline). Data are the mean \pm SE from three to four independent experiments. $\boldsymbol{B}, \boldsymbol{C}$, Bar graph representations of data in $\boldsymbol{A} . \mathbf{B}, 0 \mathrm{OCR}$ after idebenone addition with ADP and Complex I substrates present (pyruvate and malate). $\boldsymbol{C}, 0 C R$ after idebenone addition once the Complex II substrate succinate was added. ${ }^{* *} p<0.01$ relative to vehicle control; ${ }^{* * *} p<0.001$ relative to control; one-way ANOVA followed by Tukey's post hoc test.
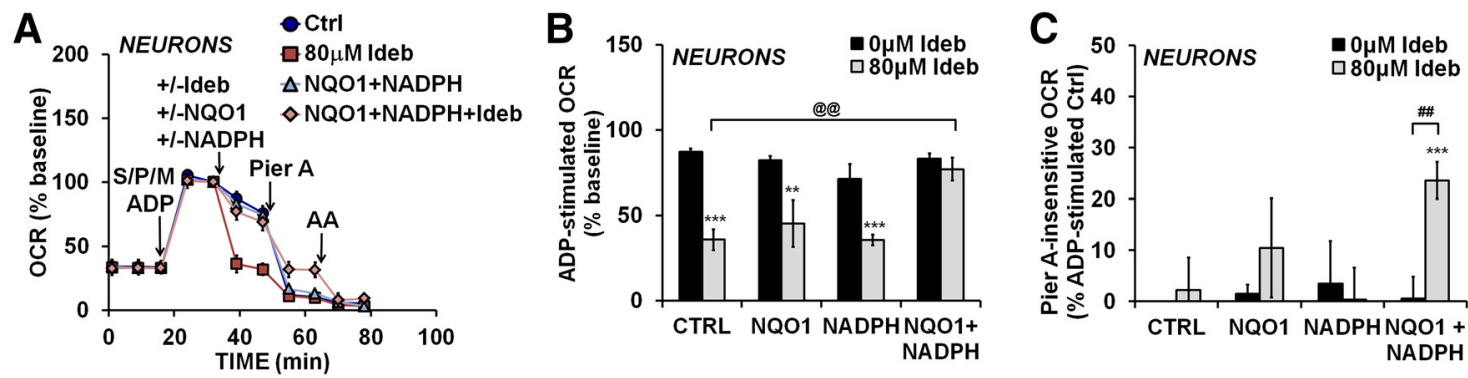

Figure 5. Delivery of recombinant NQ01 and NADPH reverses neuronal respiratory inhibition by idebenone. $A, O C R$ measurements from cortical neurons. Cells were first permeabilized with Complex I substrates present (saponin/pyruvate/malate; S/P/M), as in Figure 4A. Next, vehicle, idebenone (Ideb; $80 \mu \mathrm{m})$, NADPH (0.5 mM), and recombinant human NQ01 (15-25 U/ml) were injected either individually or in the combinations indicated. Piericidin $A$ (Pier A; $500 \mathrm{~nm}$ ) was then added to inhibit Complex I, followed by antimycin A (AA; $1 \mu \mathrm{M}$ ). OCR measurements are normalized to the point just before idebenone/vehicle addition (ADP-stimulated baseline). Data are the mean \pm SE from four to six independent experiments. $\boldsymbol{B}, \boldsymbol{C}$, Bar graph representations of data in $\boldsymbol{A}$ and including additional controls not depicted in $\boldsymbol{A}$ for clarity. $\boldsymbol{B}, O C R$ after the addition of idebenone/vehicle plus and minus the indicated treatments, expressed as a percentage of the ADP-stimulated baseline OCR. C, OCR after injection of piericidin A with and without idebenone and the indicated treatments, relative to the ADP-stimulated control (Ctrl) group (point just before piercidin A addition). .* $p<0.01$ relative to vehicle control; ${ }^{* * *} p<0.001$ relative to control; \#\#p $<0.01$ between idebenone-treated and vehicle-treated groups; @@p $<0.01$ relative to $80 \mu \mathrm{m}$ idebenone alone; two-way ANOVA followed by Tukey's post hoc test.

7.167, $p=0.0014$; Fig. $6 C$ ) and inhibited maximal respiration in neurons at all tested concentrations (one-way ANOVA: $F_{(4,17)}=$ 7.116, $p=0.0015$; Fig. $6 C$ ). MNQ induced a very slight increase in piericidin $\mathrm{A}$-insensitive respiration in neurons (one-way ANOVA: $F_{(4,17)}=3.206, p=0.03292$; Fig. $6 C$ ) that is unlikely to be biologically significant. In astrocytes, MNQ dose-dependently increased basal and piericidin A-insensitive respiration (one-way ANOVA: $F_{(4,21)}=4.599$, and $p=0.0080 ; F_{(4,21)}=5.907, p=$ 0.0024 , respectively; Fig. $6 D$ ), with no effect on maximal respiration (one-way ANOVA: $F_{(4,21)}=0.283, p=0.8857$; Fig. $6 D$ ). However, the piericidin A-insensitive OCR was completely resistant to antimycin A at MNQ concentrations $>20 \mu \mathrm{M}$, indicating that MNQ-stimulated OCR is primarily independent of the ETC (one-way ANOVA: $F_{(4,21)}=5.059, p=0.0052 ; p=0.0487$ and 0.0072 for 40 and $80 \mu \mathrm{M}$ MNQ compared with control, respectively; Fig. 6D).

Like MNQ, Me-MNQ increased the basal respiration and inhibited the maximal respiration of neurons (one-way ANOVA: $F_{(4,15)}=5.878, p=0.0047$; and $F_{(4,15)}=5.112, p=$ 0.0084 , respectively; Fig. $6 E$ ). However, Me-MNQ significantly altered basal and maximal respiration only at concentrations $\geq 40 \mu \mathrm{M} \quad(p=0.0246$ and $p=0.0283$ for $40 \mu \mathrm{M}$ Me-MNQ, respectively; and $p=0.0035$ and $p=0.0236$ for $80 \mu \mathrm{M} \mathrm{Me}$ MNQ, respectively). In astrocytes, Me-MNQ increased basal and piericidin $\mathrm{A}$-insensitive respiration in a dose-dependent manner (one-way ANOVA: $F_{(4,26)}=7.518, p=0.0004$; and $F_{(4,26)}=4.000, p=0.0117$, respectively; Fig. $\left.6 F\right)$ and had no effect on maximal respiration (one-way ANOVA: $F_{(4,26)}=$ $1.409, p=0.2588$; Fig. $6 F)$. However, Me-MNQ, when added at $40 \mu \mathrm{M}(p=0.0239)$ or $80 \mu \mathrm{M}(p=0.0155)$, caused a significant increase in antimycin A-independent OCR (one-way ANOVA: $F_{(4,26)}=5.331, p=0.0028$; Fig. $\left.6 F\right)$.

Pharmacological NQO1 induction abolishes the inhibitory effect of idebenone on cellular respiration and facilitates electron transfer to Complex III

NQO1 expression is regulated by the Nrf2-ARE antioxidant response pathway and is induced in many cell types by activators of the transcription factor Nrf2 (Li and Kong, 2009; DinkovaKostova and Talalay, 2010). We hypothesized that Nrf2 activators would reverse respiratory inhibition by idebenone in neurons and allow for bypass of Complex I-impaired respiration by enhancing NQO1 activity. We failed to increase Nrf2 or NQO1 in primary cortical neurons using several different Nrf2 activators, including sulforaphane, tert-butylhydroquinone, and carnosic acid (data not shown). However, carnosic acid treatment for $72 \mathrm{~h}$ led to a robust elevation of NQO1 in COS-7 cells, indicating that the drug was active $\left(t_{(4)}=19.33, p<0.001\right.$; Fig. 
A

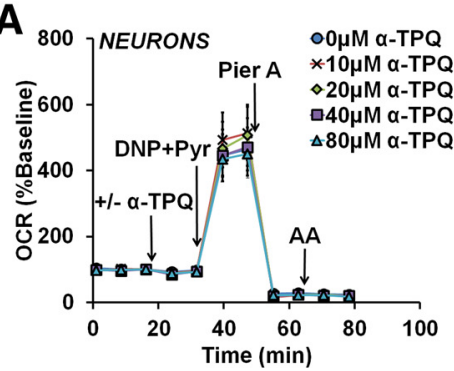

C

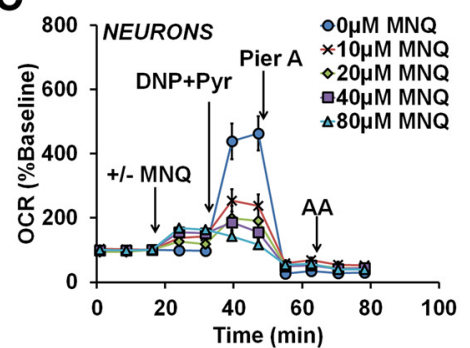

E

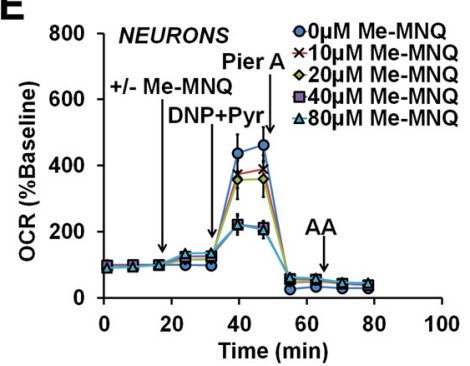

B

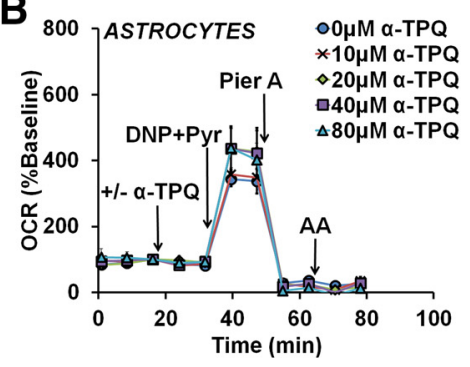

D

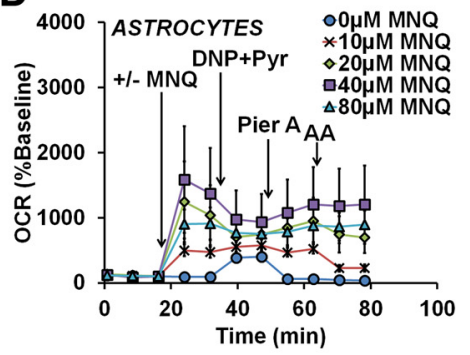

$\mathbf{F}$

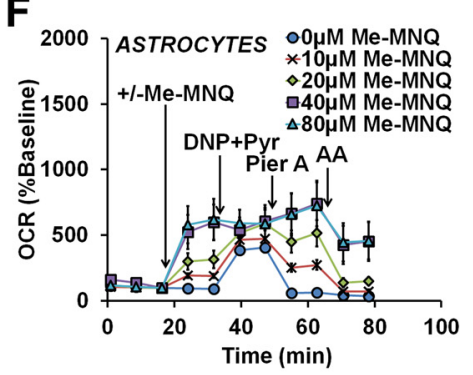

Figure 6. The Complex I bypass activity of two naphthoquinones is absent in cortical neurons that lack NQ01. $\boldsymbol{A}-\boldsymbol{F}, 0 C R$ measurements from neurons and astrocytes in response to the injection of vehicle (" $0 \mu \mathrm{m}$ ") or the indicated concentrations of alpha-tocopherolquinone ( $\alpha$-TPQ; $\boldsymbol{A}$ and $\boldsymbol{B}$ ), 2-methoxy-1,4-naphthoquinone (MNQ; $\boldsymbol{C}$ and $\boldsymbol{D}$ ), or 2-methoxy-3-methyl-1,4-naphthoquinone (Me-MNQ; $\boldsymbol{E}, \boldsymbol{F}$ ). Subsequent injections consisted of 2,4-dinitrophenol (DNP; $150 \mu \mathrm{m}$ ) + pyruvate (Pyr; $10 \mathrm{mm),} \mathrm{pierici-}$ $\operatorname{din} A($ Pier $A ; 500 \mathrm{~nm})$, and antimycin $A(A A ; 1 \mu \mathrm{M})$. Data are the mean \pm SE from three to eight independent experiments.

7A). Because basal NQO1 expression in COS-7 cells was low, we tested whether COS-7 cells could act as a surrogate for neurons to show proof-of-principle. We detected a significant interaction between carnosic acid pretreatment and idebenone treatment on the basal OCR, maximal OCR, and piericidin A-insensitive OCR of COS-7 cells $\left(F_{(3,32)}=4.828, p=0.0070 ; F_{(3,32)}=7.018\right.$, $p<0.001$; and $F_{(3,32)}=12.44, p<0.001$, respectively). As in neurons, idebenone inhibited maximal respiration in COS-7 cells that were not pretreated with carnosic acid $(p<0.001$; Fig. $7 B$, $C)$. Although idebenone supported significant piericidin Ainsensitive respiration in untreated COS-7 cells $(p=0.0070$; Fig. $7 B, D)$, the extent of this respiration was low relative to that observed in astrocytes (19.5\% compared with $59.5 \%$ of maximal OCR; compare Figs. $7 D, 1 E$ ). In carnosic acid-treated COS-7 cells, idebenone stimulated basal respiration ( $p=0.0440$ compared with control; Fig. $7 B$, first arrow), did not impair maximal respiration ( $p=0.2617$; Fig. $7 B, C)$, and greatly augmented piericidin A-insensitive oxygen consumption (to $52.2 \%$ of maximal OCR, $p<0.001$; Fig. $7 B, D$ ). Thus, the bioenergetic responses of astrocytes to idebenone were recapitulated in COS-7 cells following carnosic acid treatment. Addition of the NQO1 inhibitor dicoumarol significantly reversed these effects $[p<0.001$ for the effect on basal OCR (Fig. $7 B$, first arrow); $p=0.0053$ for maximal OCR (Fig. 7B,C); and $p<0.001$ for piericidin A-insensitive OCR (Fig. $7 B, D)$ ], suggesting that carnosic acid-induced NQO1 activity is causally involved in the transformed response of COS-7 cells to idebenone.

\section{Discussion}

Clinical trials for idebenone in neurodegenerative disorders such as Alzheimer's disease (Gutzmann and Hadler, 1998; Thal et al., 2003) and Friedreich's ataxia (Di Prospero et al., 2007a; Lynch et al., 2010; Meier et al., 2012) have not yielded convincing positive results. Previous studies indicate that idebenone may influence cellular and mitochondrial function in different ways, depending on its oxidation/reduction state (Jaber and Polster, 2015). These include acting as an antioxidant and electron donor in its reduced form but acting as a pro-oxidant with the potential to induce opening of the permeability transition pore, a mitochondrial inner membrane channel linked to cell death, in its oxidized form (Haefeli et al., 2011; Giorgio et al., 2012). In this study, we identified a fundamental difference in the way idebenone influences mitochondrial function in cortical neurons compared with cortical astrocytes. Idebenone was able to act as a direct electron donor to Complex III only in astrocytes, whereas it inhibited Complex I-dependent respiration in neurons and reduced their spare respiratory capacity. The difference between astrocytes and neurons was attributable to a large disparity in expression of the idebenone-reducing enzyme NQO1. Importantly, we showed proof-of-principle that pharmacological induction of NQO1 through the Nrf2-ARE transcriptional pathway can reverse the negative bioenergetic effects of idebenone while promoting the potentially cytoprotective Complex I bypass mechanism.

There is evidence that several different enzymes can reduce idebenone, including succinate dehydrogenase (Complex II), glycerol-3-phosphate dehydrogenase, NQO1, and NQO2 (Haefeli et al., 2011; Rauchová et al., 2012). However, our data not only showed that idebenone is reduced primarily by NQO1 in astrocytes, but also revealed that cortical neurons, which lack NQO1, are unable to compensate for the absence of NQO1 by reducing idebenone via other enzymes. Notably, we demonstrated that exogenous NQO1, when added with its substrate $\mathrm{NADPH}$, is able to reverse the respiratory impairment by idebenone in neurons and induce oxygen consumption in the presence of Complex I inhibitor. Furthermore, an inhibitory effect of idebenone on maximal respiration appeared in astrocytes following the addition of the NQO1 antagonist dicoumarol, mirroring the effect of idebenone observed in NQO1-deficient neurons.

Prior evidence suggests that idebenone impairs Complex I activity only when in the oxidized quinone form by competitively occupying the CoQ binding site (Degli Esposti et al., 1996; King et al., 2009). Thus, idebenone reduction by NQO1 appears to both allow idebenone to act as a Complex III electron donor and to eliminate Complex I inhibition by removing the oxidized form of the quinone. These findings led us to predict that 
pharmacological induction of NQO1 in neurons by activation of the Nrf2-ARE pathway would abolish inhibition of Complex I-dependent respiration by idebenone and enable direct electron transfer to Complex III.

We were unable to upregulate NQO1 protein in cortical neurons using several known Nrf2-ARE activators, likely due to epigenetic repression of $\mathrm{Nrf} 2$ in mature neurons (Bell et al., 2015). Carnosic acid was found to activate Nrf2 in COS-7 cells, among several cell types (Satoh et al., 2008). As COS-7 express little NQO1, we used these cells to establish proof-of-principle that induction of NQO1 can unleash the Complex I bypass activity of idebenone in cells in which it otherwise lacks activity. We found that the effect of idebenone on mitochondrial respiration in COS-7 cells was phenotypically similar to that observed in neurons, namely, inhibition of respiratory capacity. In contrast, cells pretreated with carnosic acid were phenotypically comparable to astrocytes, exhibiting preserved respiratory capacity and robust Complex I inhibitor-insensitive respiration. As predicted, carnosic acid treatment led to strong upregulation of NQO1 enzyme. NQO1 inhibition reversed the effect of carnosic acid treatment on the bioenergetic response to idebenone, shifting the astrocyte-like phenotype back to one resembling neurons. In sum, these results showed that pharmacological NQO1 upregulation mitigates the inhibitory action of idebenone on respiratory capacity and induces the Complex I bypass mechanism mediated by the reduced form, idebenol.

Despite our finding that idebenone reduces neuronal respiratory capacity, idebenone doses up to $75 \mathrm{mg} / \mathrm{kg}$, which yields $\sim 30 \mu \mathrm{M}$ idebenone in plasma, are safe in people (Di Prospero et al., 2007b). Although idebenone is rapidly metabolized by the liver, rodent experiments using ${ }^{14} \mathrm{C}$-labeled drug established the presence of unmetabolized idebenone in the brain within $15 \mathrm{~min}$ of administration (Torii et al., 1985; Nagai et al., 1989). Extrapolating from these studies, the concentration of idebenone in human cortex is likely $\sim 0.5 \mu \mathrm{M}$. However, idebenone is hydrophobic and concentrates in membranes. In rats, ${ }^{14} \mathrm{C}$-idebenone was enriched in the mitochondrial fraction (Torii et al., 1985) and oral drug administration enhanced the phosphorylating respiration of isolated brain mitochondria measured ex vivo (Sugiyama and Fujita, 1985). In addition, both idebenone and a major metabolite, QS-10, displayed the ability to rescue oxygen consumption in vivo by zebrafish embryos exposed to the Complex I inhibitor rotenone (Giorgio et al., 2018). Furthermore, QS-10 did not share the negative properties of oxidized idebenone (e.g., the ability to induce permeability transition; Giorgio et al., 2018). These findings support the possibility that mitochondria are a site of idebenone action in vivo.

Encouragingly, structural modifications of idebenone and library screens for compounds with Complex I bypass activity have already identified compounds with a more potent ability to rescue mitochondrial function and cellular viability (Duveau et al., 2010; Fash et al., 2013; Vafai et al., 2016; Woolley et al., 2019). Here, we found that relative to idebenone, the naphthoquinone Me-MNQ exhibited a similar ability to bypass Complex I, but at substantially lower concentrations (Fig. 6F). Recently, others have identified redox-active naphthoquinone derivatives that were superior to idebenone in their ability to rescue ATP levels in Complex I inhibitor-treated cells (Woolley et al., 2019). Hence, it is plausible that the direct electron transfer function of idebenone and other quinones can be harnessed in vivo. Unfortunately, we saw the Complex I bypass activity of MeMNQ in cortical astrocytes but not neurons, as was the case for idebenone. This finding suggests that the bypass activity of naphthoquinone derivatives may also require NQO1-reducing activity, as MNQ is a preferred NQO1 substrate (Ravasz et al., 2018). Unlike idebenone, we found that the naphthoquinones MNQ and Me-MNQ increase antimycin A-insensitive oxygen consumption, likely due to superoxide generation, which may limit their therapeutic potential.

The beneficial effect of idebenone to LHON patients and the detection of NQO1 immunoreactivity in mouse retinal ganglion cells (Heitz et al., 2012; Smith et al., 2016) suggest that, in contrast to the cortical neurons investigated in our study, some neurons are able to reduce idebenone to idebenol effectively. Interestingly, neuronal NQO1 expression was observed in the 
brains of patients with Alzheimer's disease (Raina et al., 1999; Wang et al., 2000; SantaCruz et al., 2004) and Parkinson's disease (van Muiswinkel et al., 2004). Thus, although little to no NQO1 is expressed by most neurons of the healthy brain, disease-associated changes may overcome the epigenetic repression of $\mathrm{Nrf} 2$, enabling activation of the antioxidant response pathway in stressed cells. Importantly, such cells may regain the ability to respond to Nrf2-ARE activators, enabling further elevation of NQO1 to levels where idebenone or analogs become effective. Multiple studies have demonstrated a protective effect of Nrf2 overexpression in neurons (Xiong et al., 2015; Skibinski et al., 2017), indicating that although Nrf2 expression is normally repressed in mature neurons, its induction is tolerated and can be beneficial.

In this study, we demonstrated synergism at the mitochondrial functional level between idebenone and carnosic acid. Like idebenone, carnosic acid crosses the blood-brain barrier and is nontoxic to healthy animals (Romo et al., 2013; Miller et al., 2015). Treatment with carnosic acid alone shortly after controlled cortical impact traumatic brain injury to mice improved the function of nonsynaptic brain mitochondria assessed ex vivo (Miller et al., 2015). Combining idebenone with carnosic acid treatment may have further protective potential by promoting NQO1-dependent mitochondrial bioenergetic rescue in cells competent to activate the Nrf2-ARE pathway.

Finally, while mitochondrial bioenergetic rescue in neurons is an important goal, the potential for targeting astrocytes in neurodegenerative diseases should not be overlooked. Aberrant astrocyte phenotypes have been described in multiple neurodegenerative disease states, including amyotrophic lateral sclerosis and Alexander disease (Liddelow et al., 2017; Verkhratsky et al., 2019). Our finding that idebenone promotes mitochondrial bioenergetic function in cultured cortical astrocytes, but not in neurons, suggests that idebenone may be more effective in diseases in which astrocytes contribute significantly to pathology.

Although much work is still necessary, our findings hint at why idebenone has not lived up to its initial promise as a neurotherapeutic agent-it may behave as a prodrug, with insufficient neuronal idebenone-reducing activity to convert it to its active form. Importantly, our results suggest that combination therapy with NQO1-inducing drugs like carnosic acid may be a way to overcome this limitation and capitalize on the therapeutic potential of idebenone. In the long run, the discovery of drugs displaying protective synergism with idebenone in vivo could have a positive impact on how idebenone is used to treat LHON patients, as well as in the design of clinical trials for other diseases.

\section{References}

Bell KF, Al-Mubarak B, Martel MA, McKay S, Wheelan N, Hasel P, Márkus NM, Baxter P, Deighton RF, Serio A, Bilican B, Chowdhry S, Meakin PJ, Ashford ML, Wyllie DJ, Scannevin RH, Chandran S, Hayes JD, Hardingham GE (2015) Neuronal development is promoted by weakened intrinsic antioxidant defences due to epigenetic repression of Nrf2. Nat Commun 6:7066.

Brand MD, Nicholls DG (2011) Assessing mitochondrial dysfunction in cells. Biochem J 437:575.

Chinnery PF, Brown DT, Andrews RM, Singh-Kler R, Riordan-Eva P, Lindley J, Applegarth DA, Turnbull DM, Howell N (2001) The mitochondrial ND6 gene is a hot spot for mutations that cause Leber's hereditary optic neuropathy. Brain 124:209-218.

Clerc P, Polster BM (2012) Investigation of mitochondrial dysfunction by sequential microplate-based respiration measurements from intact and permeabilized neurons. PLoS One 7:e34465.
Clerc P, Young CA, Bordt EA, Grigore AM, Fiskum G, Polster BM (2013) Magnesium sulfate protects against the bioenergetic consequences of chronic glutamate receptor stimulation. PLoS One 8:e79982.

Danielson SR, Held JM, Oo M, Riley R, Gibson BW, Andersen JK (2011) Quantitative mapping of reversible mitochondrial complex I cysteine oxidation in a Parkinson disease mouse model. J Biol Chem 286: 7601-7608.

Danilov CA, Fiskum G (2008) Hyperoxia promotes astrocyte cell death after oxygen and glucose deprivation. Glia 56:801-808.

Degli Esposti M, Ngo A, Ghelli A, Benelli B, Carelli V, McLennan H, Linnane AW (1996) The interaction of Q analogs, particularly hydroxydecyl benzoquinone (idebenone), with the respiratory complexes of heart mitochondria. Arch Biochem Biophys 330:395-400.

Dinkova-Kostova AT, Talalay P (2010) NAD(P)H:quinone acceptor oxidoreductase 1 (NQO1), a multifunctional antioxidant enzyme and exceptionally versatile cytoprotector. Arch Biochem Biophys 501:116-123.

Di Prospero NA, Baker A, Jeffries N, Fischbeck KH (2007a) Neurological effects of high-dose idebenone in patients with Friedreich's ataxia: a randomised, placebo-controlled trial. Lancet Neurol 6:878-886.

Di Prospero NA, Sumner CJ, Penzak SR, Ravina B, Fischbeck KH, Taylor JP (2007b) Safety, tolerability, and pharmacokinetics of high-dose idebenone in patients with Friedreich ataxia. Arch Neurol 64:803-808.

Duveau DY, Arce PM, Schoenfeld RA, Raghav N, Cortopassi GA, Hecht SM (2010) Synthesis and characterization of mitoQ and idebenone analogues as mediators of oxygen consumption in mitochondria. Bioorg Med Chem 18:6429-6441.

Fash DM, Khdour OM, Sahdeo SJ, Goldschmidt R, Jaruvangsanti J, Dey S, Arce PM, Collin VC, Cortopassi GA, Hecht SM (2013) Effects of alkyl side chain modification of coenzyme Q10 on mitochondrial respiratory chain function and cytoprotection. Bioorg Med Chem 21:2346-2354.

Gerencser AA, Neilson A, Choi SW, Edman U, Yadava N, Oh RJ, Ferrick DA, Nicholls DG, Brand MD (2009) Quantitative microplate-based respirometry with correction for oxygen diffusion. Anal Chem 81:68686878.

Giorgio V, Petronilli V, Ghelli A, Carelli V, Rugolo M, Lenaz G, Bernardi P (2012) The effects of idebenone on mitochondrial bioenergetics. Biochim Biophys Acta 1817:363-369.

Giorgio V, Schiavone M, Galber C, Carini M, Da Ros T, Petronilli V, Argenton F, Carelli V, Acosta Lopez MJ, Salviati L, Prato M, Bernardi P (2018) The idebenone metabolite QS10 restores electron transfer in complex I and coenzyme Q defects. Biochim Biophys Acta Bioenerg 1859:901-908

Gueven N, Woolley K, Smith J (2015) Border between natural product and drug: comparison of the related benzoquinones idebenone and coenzyme Q10. Redox Biol 4:289-295.

Gutzmann H, Hadler D (1998) Sustained efficacy and safety of idebenone in the treatment of Alzheimer's disease: update on a 2-year double-blind multicentre study. J Neural Transm Suppl 54:301-310.

Gutzmann H, Kuhl KP, Hadler D, Rapp MA (2002) Safety and efficacy of idebenone versus tacrine in patients with Alzheimer's disease: results of a randomized, double-blind, parallel-group multicenter study. Pharmacopsychiatry 35:12-18.

Haefeli RH, Erb M, Gemperli AC, Robay D, Courdier F, I, Anklin C, Dallmann R, Gueven N (2011) NQO1-dependent redox cycling of idebenone: effects on cellular redox potential and energy levels. PLoS One 6: e17963.

Heidari MM, Houshmand M, Hosseinkhani S, Nafissi S, Khatami M (2009) Complex I and ATP content deficiency in lymphocytes from Friedreich's ataxia. Can J Neurol Sci 36:26-31.

Heitz FD, Erb M, Anklin C, Robay D, Pernet V, Gueven N (2012) Idebenone protects against retinal damage and loss of vision in a mouse model of Leber's hereditary optic neuropathy. PLoS One 7:e45182.

Jaber S, Polster BM (2015) Idebenone and neuroprotection: antioxidant, prooxidant, or electron carrier? J Bioenerg Biomembr 47:111-118.

Jaber SM, Bordt EA, Bhatt NM, Lewis DM, Gerecht S, Fiskum G, Polster BM (2018) Sex differences in the mitochondrial bioenergetics of astrocytes but not microglia at a physiologically relevant brain oxygen tension. Neurochem Int 117:82-90.

James AM, Smith RA, Murphy MP (2004) Antioxidant and prooxidant properties of mitochondrial Coenzyme Q. Arch Biochem Biophys 423: $47-56$. 
Keeney PM, Xie J, Capaldi RA, Bennett JP Jr (2006) Parkinson's disease brain mitochondrial complex I has oxidatively damaged subunits and is functionally impaired and misassembled. J Neurosci 26:5256-5264.

King MS, Sharpley MS, Hirst J (2009) Reduction of hydrophilic ubiquinones by the flavin in mitochondrial NADH:ubiquinone oxidoreductase (complex I) and production of reactive oxygen species. Biochemistry 48:20532062.

Klopstock T, Yu-Wai-Man P, Dimitriadis K, Rouleau J, Heck S, Bailie M, Atawan A, Chattopadhyay S, Schubert M, Garip A, Kernt M, Petraki D, Rummey C, Leinonen M, Metz G, Griffiths PG, Meier T, Chinnery PF (2011) A randomized placebo-controlled trial of idebenone in Leber's hereditary optic neuropathy. Brain 134:2677-2686.

Laird MD, Clerc P, Polster BM, Fiskum G (2013) Augmentation of normal and glutamate-impaired neuronal respiratory capacity by exogenous alternative biofuels. Transl Stroke Res 4:643-651.

Li W, Kong AN (2009) Molecular mechanisms of Nrf2-mediated antioxidant response. Mol Carcinog 48:91-104.

Liddelow SA, Guttenplan KA, Clarke LE, Bennett FC, Bohlen CJ, Schirmer L, Bennett ML, Münch AE, Chung W-S, Peterson TC, Wilton DK, Frouin A, Napier BA, Panicker N, Kumar M, Buckwalter MS, Rowitch DH, Dawson VL, Dawson TM, Stevens B, et al., (2017) Neurotoxic reactive astrocytes are induced by activated microglia. Nature 541:481-487.

Lin MT, Beal MF (2006) Mitochondrial dysfunction and oxidative stress in neurodegenerative diseases. Nature 443:787-795.

Lynch DR, Perlman SL, Meier T (2010) A phase 3, double-blind, placebocontrolled trial of idebenone in Friedreich ataxia. Arch Neurol 67:941947.

Lynch DR, Willi SM, Wilson RB, Cotticelli MG, Brigatti KW, Deutsch EC, Kucheruk O, Shrader W, Rioux P, Miller G, Hawi A, Sciascia T (2012) A0001 in Friedreich ataxia: biochemical characterization and effects in a clinical trial. Mov Disord 27:1026-1033.

Lyseng-Williamson KA (2016) Idebenone: a review in Leber's hereditary optic neuropathy. Drugs 76:805-813.

Meier T, Perlman SL, Rummey C, Coppard NJ, Lynch DR (2012) Assessment of neurological efficacy of idebenone in pediatric patients with Friedreich's ataxia: data from a 6-month controlled study followed by a 12-month open-label extension study. J Neurol 259:284-291.

Miller DM, Singh IN, Wang JA, Hall ED (2015) Nrf2-ARE activator carnosic acid decreases mitochondrial dysfunction, oxidative damage and neuronal cytoskeletal degradation following traumatic brain injury in mice. Exp Neurol 264:103-110.

Nagai Y, Yoshida K, Narumi S, Tanayama S, Nagaoka A (1989) Brain distribution of idebenone and its effect on local cerebral glucose utilization in rats. Arch Gerontol Geriatr 8:257-272.

Nagaoka A, Suno M, Shibota M, Kakihana M (1989) Effects of idebenone on neurological deficits, local cerebral blood flow, and energy metabolism in rats with experimental cerebral ischemia. Arch Gerontol Geriatr 8:193202.

Nicholls DG, Ferguson SJ (2013) Bioenergetics 4, Ed 4. London: Academic.

Raina AK, Templeton DJ, Deak JC, Perry G, Smith MA (1999) Quinone reductase (NQO1), a sensitive redox indicator, is increased in Alzheimer's disease. Redox Rep 4:23-27.

Ratan RR, Murphy TH, Baraban JM (1994) Oxidative stress induces apoptosis in embryonic cortical neurons. J Neurochem 62:376-379.

Rauchová H, Vokurková M, Drahota Z (2012) Idebenone-induced recovery of glycerol-3-phosphate and succinate oxidation inhibited by digitonin. Physiol Res 61:259-265.

Ravasz D, Kacso G, Fodor V, Horvath K, Adam-Vizi V, Chinopoulos C (2018) Reduction of 2-methoxy-1,4-naphtoquinone by mitochondriallylocalized Nqo1 yielding $\mathrm{NAD}(+)$ supports substrate-level phosphorylation during respiratory inhibition. Biochim Biophys Acta Bioenerg 1859:909-924.

Romo VM, Garcia VR, Larrosa M, Yanez-Gascon MJ, Fromentin E, Flanagan J, Roller M, Tomas-Barberan FA, Espin JC, Garcia-Conesa MT (2013) Bioavailability of the major bioactive diterpenoids in a rosemary extract: metabolic profile in the intestine, liver, plasma, and brain of Zucker rats. Mol Nutr Food Res 57:1834-1846.

Ruas JS, Siqueira-Santos ES, Amigo I, Rodrigues-Silva E, Kowaltowski AJ, Castilho RF (2016) Underestimation of the maximal capacity of the mitochondrial electron transport system in oligomycin-treated cells. PLoS One 11:e0150967.
Safiulina D, Kaasik A, Seppet E, Peet N, Zharkovsky A, Seppet E (2004) Method for in situ detection of the mitochondrial function in neurons. J Neurosci Methods 137:87-95.

Salehi MH, Kamalidehghan B, Houshmand M, Yong MG, Sadeghizadeh M, Aryani O, Nafissi S (2014) Gene expression profiling of mitochondrial oxidative phosphorylation (OXPHOS) complex I in Friedreich ataxia (FRDA) patients. PLoS ONE 9:e94069.

SantaCruz KS, Yazlovitskaya E, Collins J, Johnson J, DeCarli C (2004) Regional NAD(P)H:quinone oxidoreductase activity in Alzheimer's disease. Neurobiol Aging 25:63-69.

Satoh T, Kosaka K, Itoh K, Kobayashi A, Yamamoto M, Shimojo Y, Kitajima C, Cui J, Kamins J, Okamoto S, Izumi M, Shirasawa T, Lipton SA (2008) Carnosic acid, a catechol-type electrophilic compound, protects neurons both in vitro and in vivo through activation of the Keap1/Nrf2 pathway via S-alkylation of targeted cysteines on Keap1. J Neurochem 104:11161131.

Schapira AH, Cooper JM, Dexter D, Clark JB, Jenner P, Marsden CD (1990) Mitochondrial complex I deficiency in Parkinson's disease. J Neurochem 54:823-827.

Schuh RA, Clerc P, Hwang H, Mehrabian Z, Bittman K, Chen H, Polster BM (2011) Adaptation of microplate-based respirometry for hippocampal slices and analysis of respiratory capacity. J Neurosci Res 89:19791988.

Schultzberg M, Segura-Aguilar J, Lind C (1988) Distribution of DT diaphorase in the rat brain: biochemical and immunohistochemical studies. Neuroscience 27:763-776.

Skibinski G, Hwang V, Ando DM, Daub A, Lee AK, Ravisankar A, Modan S, Finucane MM, Shaby BA, Finkbeiner S (2017) Nrf2 mitigates LRRK2and $\alpha$-synuclein-induced neurodegeneration by modulating proteostasis. Proc Natl Acad Sci U S A 114:1165-1170.

Smith TG, Seto S, Ganne P, Votruba M (2016) A randomized, placebo-controlled trial of the benzoquinone idebenone in a mouse model of OPA1related dominant optic atrophy reveals a limited therapeutic effect on retinal ganglion cell dendropathy and visual function. Neuroscience 319:92106.

Srinivas Bharath MM (2017) Post-translational oxidative modifications of mitochondrial complex I (NADH: ubiquinone oxidoreductase): implications for pathogenesis and therapeutics in human diseases. J Alzheimers Dis 60:S69-S86.

Stoica BA, Movsesyan VA, Knoblach SM, Faden AI (2005) Ceramide induces neuronal apoptosis through mitogen-activated protein kinases and causes release of multiple mitochondrial proteins. Mol Cell Neurosci 29:355371.

Stringer JL, Gaikwad A, Gonzales BN, Long DJ Jr, Marks LM, Jaiswal AK (2004) Presence and induction of the enzyme NAD $(\mathrm{P}) \mathrm{H}$ : quinone oxidoreductase 1 in the central nervous system. J Comp Neurol 471:289297.

Sugiyama Y, Fujita T (1985) Stimulation of the respiratory and phosphorylating activities in rat brain mitochondria by idebenone (CV-2619), a new agent improving cerebral metabolism. FEBS Lett 184:48-51.

Thal LJ, Grundman M, Berg J, Ernstrom K, Margolin R, Pfeiffer E, Weiner MF, Zamrini E, Thomas RG (2003) Idebenone treatment fails to slow cognitive decline in Alzheimer's disease. Neurology 61:1498-1502.

Tomono Y, Hasegawa J, Seki T, Motegi K, Morishita N (1986) Pharmacokinetic study of deuterium-labelled coenzyme Q10 in man. Int J Clin Pharmacol Ther Toxicol 24:536-541.

Torii H, Yoshida K, Kobayashi T, Tsukamoto T, Tanayama S (1985) Disposition of idebenone (CV-2619), a new cerebral metabolism improving agent, in rats and dogs. J Pharmacobiodyn 8:457-467.

Vafai SB, Mevers E, Higgins KW, Fomina Y, Zhang J, Mandinova A, Newman D, Shaw SY, Clardy J, Mootha VK (2016) Natural product screening reveals naphthoquinone complex I bypass factors. PLoS One 11:e0162686.

van Muiswinkel FL, de Vos RA, Bol JG, Andringa G, Jansen Steur EN, Ross D, Siegel D, Drukarch B (2004) Expression of NAD(P)H:quinone oxidoreductase in the normal and Parkinsonian substantia nigra. Neurobiol Aging 25:1253-1262.

Verkhratsky A, Ho MS, Vardjan N, Zorec R, Parpura V (2019) General pathophysiology of astroglia. Adv Exp Med Biol 1175:149-179.

Wang Y, Santa-Cruz K, DeCarli C, Johnson JA (2000) NAD(P)H:quinone oxidoreductase activity is increased in hippocampal pyramidal neurons of patients with Alzheimer's disease. Neurobiol Aging 21:525-531. 
Woolley KL, Nadikudi M, Koupaei MN, Corban M, McCartney P, Bissember AC, Lewis TW, Gueven N, Smith JA (2019) Amide linked redox-active naphthoquinones for the treatment of mitochondrial dysfunction. Medchemcomm 10:399-412.

Wu M, Neilson A, Swift AL, Moran R, Tamagnine J, Parslow D, Armistead S, Lemire K, Orrell J, Teich J, Chomicz S, Ferrick DA (2007) Multiparameter metabolic analysis reveals a close link between attenuated mitochondrial bioenergetic function and enhanced glycolysis dependency in human tumor cells. Am J Physiol Cell Physiol 292:C125C136.

Xiong W, MacColl Garfinkel AE, Li Y, Benowitz LI, Cepko CL (2015) NRF2 promotes neuronal survival in neurodegeneration and acute nerve damage. J Clin Invest 125:1433-1445.
Yakovlev AG, Ota K, Wang G, Movsesyan V, Bao WL, Yoshihara K, Faden AI (2001) Differential expression of apoptotic protease-activating factor- 1 and caspase- 3 genes and susceptibility to apoptosis during brain development and after traumatic brain injury. J Neurosci 21: 7439-7446.

Yamada K, Tanaka T, Han D, Senzaki K, Kameyama T, Nabeshima T (1999) Protective effects of idebenone and alpha-tocopherol on beta-amyloid(1-42)-induced learning and memory deficits in rats: implication of oxidative stress in beta-amyloid-induced neurotoxicity in vivo. Eur J Neurosci 11:83-90.

Zielke HR, Tildon JT, Landry ME, Max SR (1990) Effect of 8-bromo-cAMP and dexamethasone on glutamate metabolism in rat astrocytes. Neurochem Res 15:1115-1122. 